\title{
Improving doctor-patient communication in a primary care setting
}

\author{
Oleksii KORZH, Olga TSODIKOVA
}

Kharkiv Medical Academy of Postgraduate Education, Department of General Practice-Family Medicine, Ukraine

\begin{abstract}
Background. The benefits of effective communication include good working relationships and increased patient satisfaction. Effective communication can improve patient understanding of treatment, improve compliance, and lead to better health.

Aim. This literature review was conducted to gather knowledge and concepts related to doctor-patient communication and how they affect patient satisfaction and perceived quality of medical services in primary care.

Method. Various electronic databases were used to search for articles, including PubMed, Cochrane, Scopus and Google Scholar. The headings of related articles were selected, which were narrowed down before annotating the articles concerned.

Results. All the reviewed articles showed that the community was positively related to patient satisfaction and perceived quality of medical services; in both extended and developing countries. Communication creates trust that helps an independent physician to provide the best care. Conduct more visits, visit patients, encourage follow-up questions after visits, actively listen to patients and begin conversations, you can significantly improve the level of communication, level of trust and level of help. Joint decision making leads to better management and better chances of adherence to treatment.

Conclusion. Evidence suggests that a patient-centered approach with effective communication skills and trust leads to better management and increased patient satisfaction. This is associated with better adherence to treatment, better health outcomes and better perception of the quality of medical services.
\end{abstract}

Keywords: communication, communication skills, education

\section{BACKGROUND}

Good communication skills are an integral part of medical and other medical practices. Communication is important not only for professional interaction with the patient, but also for medical personnel. The benefits of effective communication include good working relationships and increased patient satisfaction. Effective communication can improve patient understanding of treatment, improve compliance, and lead to better health. It can also make pro- fessional-patient relationships fairer. Undoubtedly, however, there are obstacles to effective communication, ranging from personal relationships and ending with the limitations imposed on doctors by the organizational structures in which they work $(1,2)$.

Over the past few decades, public expectations from primary care physicians have increased, and most of them are familiar with their rights in the health care system. As a consequence, it is of paramount importance that family doctors have effective communication 
skills. It was well documented that the relationship between doctor and patient is crucial for the provision of high-quality medical care. Skillful communication helps family doctors establish relationships, solicit and share important information, and work effectively with patients, family members and the public. It was shown that this affects patient satisfaction, reduces the use of painkillers, reduces the length of hospital stay, improves recovery after surgery and a number of other biological, psychological and social results (3).

It was shown that patients immediately forget about $40-80 \%$ of the medical information provided to them by medical workers. In particular, patients with low health literacy have great difficulty in obtaining medical information. A person's medical literacy refers to his ability to receive, understand, and use information to make effective decisions about his health and health care. Health literacy is influenced by individual factors, such as educational level or cultural level, as well as the health environment, from policies and processes to communication with health care providers.

\section{THE ROLE OF COMMUNICATION IN THE PRIMARY CARE CONSULTATION}

Physicians are required to communicate verbally and in writing with a number of specialists, managers, patients, families and caregivers. Just admitting the need for good communication skills is not enough; health professionals should actively strive to achieve good communication skills, assessing their own abilities. Providers of education services must ensure that physicians have appropriate and effective training opportunities to develop and improve such skills in order to facilitate interaction with patients and others [5].

The benefits of good communication can be defined for both doctors and patients.

\section{Benefits for patients}

- The relationship between doctor and patient is improving. The physician can better search for relevant information and recognize patient problems through interaction and attentive hearing. As a result, patient problems can be more accurately determined (4).

- Good communication helps the patient to remember information and follow treatment instructions, which increases patient satisfaction $(2,3)$.
- Good communication can improve patient health and results. Better communication and dialogue through repetition and repetition between the physician and the patient has a beneficial effect in terms of improving emotional health, resolving symptoms and controlling pain (1).

- The overall quality of care can be improved by ensuring that the opinions and wishes of patients are taken into account as a reciprocal process when making decisions.

- Good communication can reduce the frequency of clinical errors (5).

\section{Benefits for doctors}

- Effective communication skills can help doctors cope with the difficulties encountered in this emotionally demanding profession. It is believed that problem communication with patients contributes to emotional exhaustion and low personal performance of doctors, as well as high psychological morbidity (10). The ability to communicate competently can also increase job satisfaction.

- Patients are less likely to complain if doctors communicate well. Thus, the likelihood that doctors will be sued is reduced.

In all interactions between the doctor and the patient, different communication skills are required at different stages of the consultation. At the beginning of the consultation, doctors must establish mutual understanding and determine the reasons for the consultation. They must collect information, structure counseling, build relationships and provide relevant information. In health care, there is a tendency to increase the need for strong communication skills in medicine. With regard to communication with patients, increasing attention to joint decision making and risk communication are two of the most important factors. For example, communication skills can help medical personnel explain the results of epidemiological studies or clinical trials to individual patients in such a way as to help patients understand the risk. Doctors can do this more effectively if they develop relationships with their patients and take into account knowledge and perceptions about the risks to public health.

Patients find themselves in a vulnerable position when they believe that medical professionals will take care of their interests. For their own good, patients should trust physicians with 
their personal information and information necessary for their proper management. Trust in the medical field can be viewed as institutional trust or interpersonal trust. With regard to the health care system, interpersonal trust is created, maintained or undermined in person with health care providers. As for institutional trust, it is placed on the medical system or institution. The interaction between the doctor and the patient is influenced by both the individual and the social systems, and trust arises within the framework of the interaction influenced by both the individual and the social systems.

Recent studies show that poor communication between medical personnel and patients is still too common. Medical professional organizations emphasized the importance of communication skills and developed various approaches to learning communication skills.

Effective communication between health professionals and patients can improve the health outcomes of both low and high medical literacy patients. Breaking the link in the chain of care is a leading factor in preventable disability and death and is a common cause of complaints about health care workers.

It has been shown that the doctor's attitude towards his patients, his ability to identify and respect patients' problems, provide relevant information and demonstrate empathy and develop patient confidence are key factors determining good adherence to medical treatment in patients. In addition, training physicians to improve their communication skills can potentially be cost-effective, as it increases adherence, which in turn improves the overall health of patients.

\section{THE SKILLS REQUIRED FOR GOOD COMMUNICATION}

There is not just one way that doctors need to communicate in order to be effective, but there are several recommendations. Developing these skills on an individual basis is when communication becomes an art. Each doctor may know the recommendations, but the development and use of these skills in his consultations is a constant journey and process of improving this art throughout his life. Communication guidelines emphasize the importance of verbal and non-verbal communication skills in developing a common understanding. Open, attentive posture, eye contact, active listening, and encouraging the patient to speak with clues such as nods are all important non-verbal communication skills. Verbal cues can include starting a consultation, prompting the patient to tell their story without interruption, and then ask additional open questions to continue to identify additional problems or problems.

If all questions are clarified, this can eliminate the need for unnecessary research and identify the psychological causes of physical symptoms. It may also prevent the patient from returning with unresolved problems. The importance of listening rather than interruption has been shown to increase the likelihood of detecting mental illness. After all questions have been clarified, to ensure a common understanding, it is important to summarize and reflect what was heard.

Empathy is another important skill. Empathy contributes to the professional satisfaction of the clinician and provides more effective care, as patients are likely to be open with a sympathetic doctor. Examining the patient's perspective, developing trust and understanding the patient's history and psychosocial health factors are essential elements of care. Using these skills will ensure good communication and will promote an effective and therapeutic relationship between the patient and the doctor.

Much of the medical literature is devoted to the study of the skills necessary for open and effective communication with patients. Toronto's consensus statement on patient-to-doctor communication was published more than 20 years ago. This statement was based on research collected from the 1970s, and led to the formation of a patient-oriented educational tool, which is now in the second edition and is widely used in medical education5. Patient-oriented treatment explores the problems of patients, seeks to understand the person as a whole and their experience of the disease, to promote general understanding, to prevent or manage health problems and improve the patient's current relationship with the doctor [6].

The Calgary-Cambridge guide contains a curriculum for teaching the skills needed to communicate well with patients. It also contains evidence-based guidelines and highlights the benefits of more efficient and effective consultation. This manual, also frequently used in medical education, describes the necessary skills and provides the basis for clinical consultation. The required skills are divided into three types: 1) communication skills - this is what doc- 
tors report; 2) job skills - this is how doctors communicate; and 3) perceptual skills - this is what the doctor thinks and feels. The focus is on process skills, but this broad understanding of the skills needed for good communication allows for barriers to effective communication to be taken into account. Fundamental attitudes, assumptions, previous experience, personality, language barriers, level of education, and emotions influence counseling. Little has changed in the basic skills needed for good communication; However, the literature continues to strengthen our knowledge that a patient-centered communication model is useful, effective, efficient, and therapeutic in itself $(7,8)$.

The therapeutic relationship between patient and physician uses the clinician as a therapeutic intervention and is part of the art of communication. This art will develop over time and will be individualized, but has several common components. He uses an effective relationship between patient and physician to provide clinical treatment as part of a medical consultation, where the focus is on treating and treating the entire patient, rather than solving the problem. This is especially true in mental health counseling, where the focus is on a healing path that allows recovery, rather than a "broken" patient who needs a "remedy." Mental health counseling usually does not have a single clinical treatment that promotes recovery, but rather permanent therapeutic relationships in combination with evidence-based psychological strategies and, if necessary, pharmacological treatment. A qualitative study of healing relationships in primary care attempted to analyze how the relationship between patient and physician develops. This study showed that trust, hope, and a sense of fame were important for healing to occur. Despite all the technological advances of the last decades, caring, compassionate doctors remain the best therapeutic tool in medicine.

Communication has been shown to have a positive effect on patients, such as patient adherence to treatment, patient satisfaction, and the best indicator of subsequent treatment. Patients with higher confidence in their physician usually have better health behaviors, fewer symptoms, higher quality of similar and were more satisfied with the treatment. Health professionals, especially physicians, must convince their patients to share personal information, take tests and take chemicals in the form of drugs in their bodies. Trust plays a vital role to do it all.
Patient satisfaction, which is influenced by trust, is an important indicator of the quality of hospitals and the clinical services provided. Studies have shown a strong correlation between patient satisfaction and quality, including the safety of medical care $(4,7,8)$.

\section{THE SIGNIFICANCE OF EDUCATION IN COMMUNICATION SKILLS}

Continuing education in the field of communication skills is very important for undergraduate and graduate students of medical education. The ability of a student to communicate effectively is one of the core competencies that has been transformed into the necessary student learning outcomes for medical education (9).

However, for students to improve their communication skills, individual practice in a safe environment and receiving feedback is ideal. Standardized patients are useful for this purpose. A standardized patient is simply a person who behaves like a patient, but is trained so that every time he or she is interviewed, the same information is provided, followed by the same behavioral pattern. The advantage is that each intern receives the same clinical scenario so that it can be compared. For this purpose you can use an actor or other qualified volunteers. A standardized patient can give immediate feedback from the patient's point of view on the trainee. However, since a standardized patient must understand the seriousness of the goal and their importance in this process, he must be trained and smart enough to give objective, constructive feedback. Therefore, choosing the right standardized patient is an important task (10).

The emphasis on communication skills continues in the graduate school of general education.

The key principles are dignity, respect, encouraging the active participation of patients in health care decision making, communication and information sharing, as well as facilitating collaboration with patients. Improving treatment adherence, reducing morbidity and improving the quality of life of patients are among the benefits of patient-oriented care. Recently, there has also been an interest in formal training in intermedical communication in order to reduce the likelihood of medical errors and improve patient care by improving communication between doctors (20). Perhaps the next area of 
attention of medical education will be to strengthen the already significant work done. in improving communication with patients.

There is strong evidence that communication skills can be taught, especially using experimental methods. To be effective, communication skills training should include: evidence of existing communication deficiencies, their causes and consequences for patients and doctors; the evidence base for the skills needed to overcome these shortcomings, the demonstration of skills that need to be learned, the ability to practice skills in a controlled and safe environment, as well as constructive feedback on performance and thinking about the reasons (10, 11).

In Ukraine, the acquisition of communication skills between a doctor and a patient practically does not exist in any training program for undergraduate and graduate students. There is also little research in this area. Therefore, it is extremely important to conduct a comprehensive study to find out the needs of students and specialists, as well as to outline the goals and conditions for learning this skill.

Doctors who want to improve their communication efforts can easily do this with a few simple strategies. Patients want doctors to ask them questions that they find sensitive. Patient-centered care models depend on how patients and providers communicate on sensitive issues, but such conversations may not occur as often as they should. Doctors who take the initiative to ask about sensitive issues will improve their level of communication and the level of help they can provide to their patients.
Family doctors should also take the time to provide very specific, clear instructions when prescribing new drugs or sending patients for future laboratory tests and tests. Diagnoses, in particular, should be explained in detail to patients who are concerned about the next steps and their overall prognosis.

Communication creates trust that helps independent physicians provide better care. Spending more time with the patient during the visit, encouraging follow-up questions after the visit, actively listening to the patient and initiating conversations, can significantly improve the level of communication, the level of trust and the level of help.

\section{CONCLUSION}

Current literature illustrates the importance of communication between the doctor and patient for the best and effective treatment of patients. Trust is something that needs to be built and acquired, and having good communication skills helps build this trust between the doctor and the patient. Evidence shows that communication with a patient-centered approach improved patient outcomes when patients were satisfied with the services they were offered in a medical facility. This is due to better adherence to treatment and a better perception of the quality of medical services. The importance of communication between the doctor and patient is equally important for both developed and developing countries in terms of patient satisfaction and quality of medical services.

Conflict of interest: none declared Financial support: none declared

\section{REFERENCES}

1. Federman A, Sono M, Wolf M, Siu A, Halm E, 2009, Health literacy and cognitive performance in older adults, Journal of The American Geriatrics Society, vol. 57, n. 8, pp.1475-80.

2. Cegala DJ, Lenzmeier Broz S. Physician communication skill training: a review of theoretical backgrounds, objectives and skills. Med Educ 2002, 36(11):1004-1016.

3. Spencer JA \& Silverman J (2004). Communication education and assessment: taking account of diversity. Medical Education. 38: 116-8.

4. Hersh L, Salzman B, Snyderman D, 2015, 'Health literacy in primary care practice', American Family Physician, vol. 92, no. 2, pp.118-124.
5. Haun, J Patel, N French, D Campbell, R Bradham, D Lapcevic, W 2015, Association between health literacy and medical care costs in an integrated healthcare system: a regional population based study, $B M C$ Health Service Research, vol. 15, pp.249-260.

6. Banerjee A, Sanyal D (2012) Dynamics of doctor-patient relationship: A cross-sectional study on concordance, trust, and patient enablement. J Family Community Med 19: 12-19.

7. Anhang Price R, Elliott MN, Zaslavsky AM, Hays RD, Lehrman WG, et al. (2014) Examining the role of patient experience surveys in measuring health care quality. Med Care Res Rev 71: 522-554.
8. Isaac T, Zaslavsky AM, Cleary PD, Landon BE (2010) The Relationship between patients' perception of care and measures of hospital quality and safety. Health Serv Res 45: 1024-1040.

9. Tongue JR, Howard R, Forese LL (2005) Communication skills for patient-centered care. J Bone Joint Surg 87: 652-658.

10. Maguire P \& Pitceathly C (2002). Key communication skills and how to acquire them. BMJ 325: 697-700.

11. Haidet $P$, Dians JE, Paterniti DA, Hechtel L, Chang T, Tseng E, Rogers JC. Medical student attitudes toward the doctor-patient relationship. Med Educ. 2002;36:568-574. 